\title{
Biological Activities and Chemical Profiles of Selected Soil Fungi Isolated from Sirindhorn Peat Swamp Forest of Narathiwat, Thailand
}

(Aktiviti Biologi dan Profil Kimia Kulat Tanah Terpilih yang Dipencilkan dari Hutan Paya Gambut Sirindhorn di Narathiwat, Thailand)

\author{
Sukanya Dej-adisai*, Narisorn Kaewchutima, Chatchai WattanapiromsakUl \& Sathianpong Phoopha
}

\section{ABSTRACT}

A total of 54 soil fungi were collected from Sirindhorn peat swamp forest in Narathiwat Province which is the last peat swamp forests in Thailand. They were extracted using four different solvents and 104 crude extracts were obtained. The bioactivity screening of these samples was done employing alpha-glucosidase inhibition, anti-inflammation using nitric oxide inhibition and cytotoxicity using cancer cell line (MCF-7). The results showed that SPSF224 crude extract had the best biological activities in the screening tests and was then selected for further study. The SPSF224 was identified as Penicillium maximae. The crude extracts of $\mathrm{P}$. maximae were determined for chemical profile, $I C_{50}$ value of alpha-glucosidase inhibition, antioxidant and antimicrobial activities against Staphylococcus aureus, Trichophyton mentagrophytes, and $\mathrm{T}$. rubrum. This was the first report of chemical investigation and biological activity evaluation of P. maximae.

Keywords: Alpha-glucosidase inhibition; antidiabetic; anti-microbial; antioxidant; Penicillium maximae

\section{ABSTRAK}

Sebanyak 54 kulat tanah telah dikumpulkan daripada hutan paya gambut Sirindhorn di Wilayah Narathiwat yang merupakan hutan paya gambut terakhir di Thailand. Kesemuanya diekstrak menggunakan empat pelarut yang berbeza dan 104 ekstrak kasar diperoleh. Penyaringan bioaktiviti sampel ini dilakukan dengan menggunakan aktiviti perencatan alfa-glukosidase, anti-radang menggunakan perencatan nitrik oksida dan sitotoksisita menggunakan titisan sel kanser (MCF-7). Hasil kajian menunjukkan bahawa ekstrak kasar SPSF224 mempunyai aktiviti biologi terbaik dalam ujian saringan dan kemudian dipilih untuk kajian lebih lanjut. SPSF224 dikenal pasti sebagai Penicillium maximae. Ekstrak kasar P. maximae ditentukan untuk profil kimia, nilai $I C_{50}$ perencatan alfa-glukosidase, aktiviti antioksida dan antimikrob terhadap Staphylococcus aureus, Trichophyton mentagrophytes dan T. rubrum. Ini adalah laporan pertama penyelidikan kimia dan penilaian aktiviti biologi P. maximae.

Kata kunci: Antidiabetik; antimikrob; antioksidan; Penicillium maximae; perencatan alfa-glukosidase

\section{INTRODUCTION}

Diabetes mellitus (DM) is a group of metabolic diseases characterised by hyperglycemia due to the lack of insulin secretion, action or both. DM is a major noncommunicable disease or NCDs in the world. The number of people with diabetes worldwide between the ages of 20 and 79 in 2017 was 425 million peoples and will be increased to 629 million by 2045 (WHO 2019). The Bureau of Non-Communicable Diseases, Ministry of Public Health, Thailand showed the statistics of diabetic patients per 100,000 population per year in Thailand in 2007 and 2015 were 500,347 and 802,017, respectively (Bureau of NCD 2019). 
A defect in insulin production is the primary cause of rising blood sugar levels. Alpha-glucosidase enzyme is a key enzyme located at the brush border of the small intestine used for delaying blood glucose absorption which hydrolyses 1, 4 alpha-glycosidase bonds in the terminal position of the polysaccharide chain (Gao et al. 2008). The complex carbohydrates in the lumen of the small intestine have to be broken up by alpha-glucosidase before the resulting glucose molecules can be taken up and finally reach the bloodstream. Postprandial hyperglycemia can be improved by inhibition of small intestinal alpha-glucosidase activity (Hakamata et al. 2009). The drugs currently being used for hyperglycemia treatment by alpha-glucosidase inhibitory mechanism include miglitol (Kalra 2014), voglibose (Horii et al. 1986) and acarbose (Bischoff 1994) which have been initially isolated from microorganisms.

It is well known that long term DM is often associated with secondary complications due to over production of free radicals and malfunction of antioxidant process giving rise to increasing oxidative stress. From previous clinical studies, the results of antioxidant treatments showed that they could improve symptoms of diabetic polyneuropathy and increased glucose transport in skeletal muscle cells. The report also presented that the intake of antioxidant could reduce the risk of development on type 2 DM (Scott \& King 2004).

Moreover, DM patients are susceptible to be infected with pathogens such as Staphylococcus aureus, S. epidermidis, Mycobacterium tuberculosis, and Streptococcus pneumoniae because their immune systems are weak (Casqueiro \& Alves 2012). Therefore, combination treatment with antioxidant and antibacterial agents could help to improve DM treatment effectively (Fuchs et al. 2013). The antimicrobial effects of some of active ingredients of Penicillium species including griseofulvin, is one of antifungal antibiotics currently used for systematic fungal infection treatment which was isolated from P. griseofulvum (MacMillan 1954).

Fungi are found in a variety of habitats such as soil, associated with plants, water, associated with other fungi and ruminant guts (Dighton \& White 2017). Secondary metabolites produced by fungi usually show inhibitory or toxicity effects on other organisms. Accordingly, fungal metabolites have been developed to be used in pharmaceuticals (Shwab \& Keller 2008) due to their antibacterial, antifungal, anticancer (Zhao et al. 2013), anti-inflammatory (Deshmukh et al. 2009), antioxidant
(Sadananda et al. 2014) and cytotoxic activities (Turbyville et al. 2006).

Peat swamp forests are tropical moist forests and they create a thick layer of acid soil (Thawai 2004) with high relative biodiversity and humidity (Boonyuen et al. 2012). The microbes living in this area can survive in this harsh environment. The Sirindhorn Peat Swamp Forest Nature Research and Study Centre is located in one of the last remaining peat swamp forests in Thailand. This forest area is reported to spread over 224 square kilometers (ORDPB 2019). Preliminary study showed that various fungi such as Aspergillus sp., Penicillium sp., Trichoderma sp., and Gongronella sp. have been isolated from soil obtained from Sirindhorn peat swamp forest. Pharmacological activities of these fungi such as antifungal (Wang et al. 2012), cytotoxic, insecticide (Rukachaisirikul et al. 2013), antibacterial (Cazar et al. 2005), antioxidant (Trisuwan et al. 2011), antimalarial and anticancer (Trisuwan et al. 2014) have been reported. This study aimed to search for the active substances for antidiabetic activity from fungi isolated from the soil of Sirindhorn peat swamp forest by assessing alpha-glucosidase inhibitory efficacy. The effective compound or extract could serve as a lead substance for further development of anti-diabetic drug. Additionally, bioactivity screening of alpha-glucosidase inhibition, antiinflammation and cytotoxicity of the fungal crude extracts from Sirindhorn peat swamp forest are herewith reported.

\section{Materials AND Methods}

\section{FUNGAL MATERIALS}

The 54 soil fungi were collected from Sirindhorn peat swamp forest, Narathiwat, Thailand. They were isolated by the researchers from Department of Microbiology, Faculty of Science, Prince of Songkla University, Songkhla, Thailand. The isolated fungi were cultivated and extracted using four different solvents to obtain 104 sample extracts which were then screened for bioactivity determination. The bioactivity screening performed on these samples were alpha-glucosidase inhibition, antiinflammation (nitric oxide inhibition) and cytotoxicity using cancer cell line (MCF-7).

DETERMINATION OF ALPHA-GLUCOSIDASE INHIBITORY ACTIVITY

This assay was modified from previously reported 
protocol using colorimetric method in 96-well plate (Dejadisai \& Pitakbut 2015). Samples ( $2 \mathrm{mg} / \mathrm{mL}$ ) were added to $50 \mu \mathrm{L}$ of $10 \mathrm{mM}$ phosphate buffer solution $(\mathrm{pH} 7)$, which contained $2 \mathrm{mg} / \mathrm{mL}$ of bovine serum albumin and $0.2 \mathrm{mg} / \mathrm{mL}$ of sodium azide, and then incubated at $37^{\circ} \mathrm{C}$ for 2 min with $50 \mu \mathrm{L}$ of $\alpha$-glucosidase enzyme (1 unit/ $\mathrm{mL})$. The result of $\alpha$-glucosidase inhibition activity was processed using the following equations:

$$
\begin{aligned}
& \text { Velocity }=\frac{\Delta \text { Absorbance at } 405 \mathrm{~nm}}{\Delta \text { Time }} \\
& \% \text { Inhibition }=\frac{\mathrm{V} \text { control-V sample }}{\mathrm{V} \text { control }} \times 100
\end{aligned}
$$

\section{DETERMINATION OF ANTI-INFLAMMATORY ACTIVITY}

Inhibitory effect on nitric oxide (NO) production by the 104 fungal extracts $(25 \mu \mathrm{g} / \mathrm{mL})$ were carried out using RAW264.7 cells following Dej-adisai et al. (2018). NO production was determined by measuring the accumulation of nitrite in the culture supernatant using the Griess reagent. The reagent consisted of $\mathrm{N}-(1-$ naphthalenediamine) and sulfanilic acid. Under acidic conditions, sulfanilic acid (sulfanilamide) was converted by nitrite to a diazonium salt. $N$-(1-naphthalenediamine) was added into diazonium salt to form a colored azo dye (red-pink color). The optical density was measured with a $570 \mathrm{~nm}$ using microplate reader. L-nitroarginine (L-NA), indomethacin and caffeic acid phenethyl ester (CAPE) were used as positive controls.

\section{DETERMINATION OF CYTOTOXIC ACTIVITY}

Cytotoxic activity against human cancer cells for 104 fungal extract samples $(25 \mu \mathrm{g} / \mathrm{mL})$ were determined by performing sulforhodamine B (SRB) colorimetric assay. SRB assay was used for measuring the cellular protein content using two of the sulfonic groups to bind to basic amino acid in the cell under mild acid condition, which show pink color. Human cancer cells (human breast carcinoma cell line (MCF-7)) and a human normal cell (human gingival fibroblast cell line (HGF)) were used for cytotoxicity test. Camptothecin was used as a positive control. The experiment was tested in 96 well plates and detected using microplate reader at wavelength $492 \mathrm{~nm}$ (Dej-adisai et al. 2018).

\section{FUNGAL IDENTIFICATION}

Fifty-four soiled fungi were collected from Sirindhorn peat swamp forest in Narathiwat Province which is the last peat swamp forests in Thailand. They were extracted by 4 different solvents and obtained 104 crude extracts. The bioactivity screening of the 104 crude extracts showed that SPSF224 crude extract had the best biological activities. SPSF224 was selected for further species identification which was performed by Leibniz-Institut DSMZ-Deutsche Sammlungvon Mikroorganismen und Zellkulturen $\mathrm{GmbH}$, Germany. The fungus was subjected to DNA extraction and sequencing of the rDNA ITS fragment (Schoch et al. 2012). Additionally, we amplified and sequenced the large subunit of rDNA (LSU) and partial calmodulin gene (Visagie et al. 2013). The fungus rDNA-ITS fragment showed two different operons and was therefore not useful for identification. Comparison of assembled calmodulin sequences was performed with GenBank, Myco ID and various databases as well as with the type species of Penicillium maximae (GenBank Accession: KC773821). P. maximae belongs to the section Sclerotiora within the genus Penicillium (Visagie et al. 2014).

\section{FUNGAL FERMENTATION AND EXTRACTION OF SPSF224}

SPSF224 was grown on potato dextrose agar (PDA) at $25^{\circ} \mathrm{C}$ for five days. Five pieces of $0.5 \times 0.5 \mathrm{~cm}^{2}$ mycelial agar plugs were inoculated into $500 \mathrm{~mL}$ Erlenmeyer flasks containing $300 \mathrm{~mL}$ of PDA at room temperature for 21 days. The fungal culture $(60 \mathrm{~L})$ was filtered to separate into wet mycelia and the filtrate. The filtrated broth was transferred to a separatory funnel and the same volume of ethyl acetate was added each time. The organic layer was evaporated to dry under reduced pressure to provide broth ethyl acetate extract, BE (red-brown paste, $5.77 \mathrm{~g}$ ). The fungal mycelium was soaked for 3 days in methanol. The $\mathrm{MeOH}$ layer was concentrated by an evaporator and added to $150 \mathrm{~mL}$ of distilled water. The mixture was then shaken with $500 \mathrm{~mL}$ of hexane. The aqueous layer was extracted three times with an equal volume of ethyl acetate and then evaporated to obtain cell hexane extract, $\mathrm{CH}$ (yellow-brown paste, $1.35 \mathrm{~g}$ ), cell ethyl acetate extract, CE (red-brown paste, $2.47 \mathrm{~g}$ ) and cell methanol extract, CM (black-brown paste, $3.59 \mathrm{~g}$ ). Then these extracts from SPSF224 were further studied for chemical profiling and biological activities. 


\section{FUNGAL PURIFICATION}

The extract from SPSF224 BE (5.767 g) was loaded onto the quick column by dry-loading technique. Then, a gradient solvent system as a mobile phase was eluted through the column with a mixture of hexane, ethyl acetate, methanol and water from 100:0:0:0 to 0:50:40:10 to yield 19 subfractions F1 to F19. F12 (0.972 g) was further purified using classical column chromatographic technique. The separation was carried out using a mixture of chloroform and methanol from 100:0 to 50:50 to obtain 11 subfractions. F12-8 (0.121 g) was loaded on the surface of Sephadex ${ }^{\circledR}$ LH-20 column chromatography using methanol as eluent to produce 8 fractions. F128-8 (68 mg) was purified by Sephadex ${ }^{\circledR}$ LH-20 column chromatography. The same elution protocol was repeated giving 7 subfractions. Fraction F12-8-8-4 was isolated and determined as semi-purified PM1 (30.2 mg).

\section{GAS CHROMATOGRAPHY-MASS SPECTROMETRY}

Gas Chromatography-Mass Spectrometer (GC-MS) (5977A Series GC/MSD System, Agilent Technologies, USA) was used to analyse the crude extracts. The samples were chromatographed on capillary columns, size 30 $\mathrm{m} \times 250 \mu \mathrm{m} \times 0.25 \mu \mathrm{m}$. One microliter of extract was injected into an injector at $70^{\circ} \mathrm{C}$ and held for $3 \mathrm{~min}$. The temperature was increased by $15^{\circ} \mathrm{C} / \mathrm{min}$ until it reached $200{ }^{\circ} \mathrm{C}$ without holding. It was then increased with a program rate of $3{ }^{\circ} \mathrm{C} / \mathrm{min}$ to $300{ }^{\circ} \mathrm{C}$ and was held for 10 min. The identification of the components in the extract was carried out by comparison of their retention time and mass spectral fragmentation pattern with those stored in the computer library.

\section{CHEMICAL SCREENING}

The extracts from SPSF224 were further studied for its chemical profile by identification of the presence of tannin, alkaloids, steroid, triterpenoid, cardiac glycoside, saponin, anthraquinone, coumarin, and flavonoid. The chemical screening tests were performed according to previously reported methods (Farnsworth 1966; Harborne 1984; Trease \& Evans 1983) with some modifications.

\section{DETERMINATION OF TOTAL PHENOLIC CONTENT (TPC)}

Total phenolic content of $P$. maximae extracts was estimated using the Folin-Ciocalteu colorimetric assay according to the method of Singleton and Rossi (1965) with some modifications. Stock solutions of samples and standard (gallic acid) at the concentration of $5 \mathrm{mg} / \mathrm{mL}$ were dissolved in absolute ethanol. Total phenolic contents were obtained from regression equation $\mathrm{Y}=0.0101 \mathrm{X}+0.0111$ with $\mathrm{R}^{2}$ value of 0.999 and expressed as $\mathrm{mg} / \mathrm{g}$ gallic acid equivalent using the equation below:

$$
\text { Total phenolic content }(\mathrm{mg} / \mathrm{g})=\frac{c \times D F \times V}{W}
$$

where $\mathrm{C}$ is the concentration of gallic acid $(\mu \mathrm{g} / \mathrm{mL})$; $\mathrm{DF}$ is the dilution factor; $\mathrm{V}$ is the volume of fungal extract $(\mathrm{mL})$; and $\mathrm{W}$ is the weight of fungal extract (mg).

\section{DETERMINATION OF TOTAL FLAVONOID CONTENT}

The total flavonoid content of $P$. maximae extracts was determined by aluminum chloride colorimetric method as described by Chang et al. (2002) with some modifications. The sample $(500 \mu \mathrm{L}$ of $1 \mathrm{mg} / \mathrm{mL})$ was mixed well with $1.5 \mathrm{~mL}$ of $95 \%$ ethanol, $100 \mu \mathrm{L}$ of $10 \% \mathrm{AlCl}_{3}$, $100 \mu \mathrm{L}$ of $1 \mathrm{M} \mathrm{CH}_{3} \mathrm{COOK}$ and $2.8 \mathrm{~mL}$ of distilled water. Five varying concentrations of quercetin were used in preparing the standard curve. Regression equation of the curve, $\mathrm{Y}=0.0033 \mathrm{X}+0.0072$ with $\mathrm{R}^{2}$ value of 0.999 was obtained. The equation was used to calculate the quercetin content in $1 \mathrm{~g}$ of sample $(\mathrm{mg}$ quercetin equivalence $(\mathrm{QE}) / \mathrm{g}$ of sample).

\section{DETERMINATION OF ANTIOXIDANT ACTIVITY}

The radical scavenging activity of the various extracts of $P$. maximae were determined using 2,2-diphenyl-1picrylhydrazil free radical scavenging assay. The samples $(200 \mu \mathrm{g} / \mathrm{mL})$ or $2 \mathrm{~mL}$ of $200 \mu \mathrm{g} / \mathrm{mL}$ quercetin were mixed well with $2 \mathrm{~mL}$ of $6 \times 10^{-5} \mathrm{M}$ DPPH. Ethanol was used as blank. The mixtures were incubated for $20 \mathrm{~min}$ in the dark. Measurement of absorbance at $520 \mathrm{~nm}$ was done using UV-vis spectrometers and percentage of antioxidant activity was calculated using the following equation:

$$
\% \text { inhibition }=\left(\frac{\text { ODcontrol-ODsample }}{\text { ODcontrol }}\right) \times 100
$$

\section{DETERMINATION OF ANTIMICROBIAL ACTIVITY}

The preliminary screening of antimicrobial activity was carried out using the agar disc diffusion assay (Lorian 2005). Staphylococcus aureus (ATTC 25923), S. epidermis (TISTR 517), Propionibacterium acnes (DMTS 14916) and Methicillin-resistant S. aureus (MRSA 1350II06) were selected for Gram positive bacteria. Escherichia 
coli (ATCC35218) and Pseudomonas aeruginosa (ATCC10145) were selected for Gram negative bacteria. Candida albicans (TISTR 5779) was selected for yeast and Microsporum gypseum, Trichophyton rubrum, and T. mentagrophytes were selected for fungi. Five fungal extracts of SPSF224 were dissolved in DMSO and diluted to achieve a concentration of $200 \mu \mathrm{g} / \mathrm{mL}$ and then $10 \mu \mathrm{L}$ of samples were dropped on the sterile paper disc. All test plates were incubated for $24 \mathrm{~h}$ at $37^{\circ} \mathrm{C}$ for bacteria, 24 to $48 \mathrm{~h}$ at $35^{\circ} \mathrm{C}$ for yeast and 7 days at 30 ${ }^{\circ} \mathrm{C}$ for fungi. The appearance of zone of inhibition on the plates was measured in diameter $(\mathrm{mm})$.

\section{RESULTS AND DISCUSSION}

BIOACTIVITY DETERMINATION OF FUNGAL EXTRACTS

A total of 104 crude extract samples from 54 fungi collected from Sirindhorn peat swamp forest were determined for their biological activities as anti-alpha-glucosidase, antiinflammatory and cytotoxic (Table 1). Thirty-eight samples showed anti-alpha-glucosidase activity of over $90 \%$ inhibition. Only two samples showed potential effects of anti-inflammatory and cytotoxic activities at $25 \mu \mathrm{g} / \mathrm{mL}$ which SPSF224BE and SPSF224CE. Hence, SPSF224 was selected for further studies.

TABLE 1.Bioactivity determination of 104 fungal extracts

\begin{tabular}{|c|c|c|c|c|c|}
\hline \multirow{2}{*}{ No. } & \multirow{2}{*}{ Extract } & \multirow{2}{*}{$\begin{array}{c}\text { Anti-alpha-glucosidase } \\
\text { activity } \\
\text { (\% inhibition) }\end{array}$} & \multicolumn{2}{|c|}{$\begin{array}{c}\text { Anti-inflammation } \\
\text { (\% inhibition) }\end{array}$} & \multirow{2}{*}{$\begin{array}{c}\text { Cytotoxicity on MCF-7 } \\
\text { (\% inhibition) }\end{array}$} \\
\hline & & & $\begin{array}{l}\text { Nitric oxide } \\
\text { inhibition }\end{array}$ & Cytotoxic effect & \\
\hline 1. & SPSF001BE & $43.95 \pm 3.65$ & $35.39 \pm 3.57$ & $-2.13 \pm 3.78$ & $18.88 \pm 5.36$ \\
\hline 2. & SPSF002BE & $27.80 \pm 3.73$ & $43.63 \pm 2.78$ & $0.94 \pm 1.30$ & $59.42 \pm 4.08$ \\
\hline 3 & SPSF003BE & $99.71 \pm 0.29$ & $37.73 \pm 2.22$ & $1.26 \pm 4.89$ & $11.24 \pm 5.36$ \\
\hline 4. & SPSF004BE & $63.98 \pm 3.62$ & $32.22 \pm 4.55$ & $0.46 \pm 3.48$ & $9.90 \pm 5.97$ \\
\hline 5. & SPSF005BE & $18.19 \pm 4.00$ & $108.64 \pm 2.04$ & $85.23 \pm 1.71$ & $99.32 \pm 0.22$ \\
\hline 6. & SPSF006BE & $50.07 \pm 4.29$ & $56.57 \pm 3.43$ & $-2.94 \pm 2.90$ & $38.63 \pm 2.57$ \\
\hline 7. & SPSF007BE & $100.20 \pm 0.24$ & $26.24 \pm 2.80$ & $2.69 \pm 3.39$ & $39.10 \pm 1.30$ \\
\hline 8. & SPSF008BE & $99.55 \pm 0.08$ & $23.42 \pm 3.15$ & $1.12 \pm 1.04$ & $11.78 \pm 5.60$ \\
\hline 9. & SPSF009BE & $99.75 \pm 0.27$ & $33.71 \pm 2.37$ & $3.56 \pm 1.95$ & $-3.90 \pm 5.39$ \\
\hline 10. & SPSF010BE & $7.18 \pm 5.33$ & $22.95 \pm 1.21$ & $3.86 \pm 0.92$ & $66.00 \pm 4.00$ \\
\hline 11. & SPSF001BE & $43.95 \pm 3.65$ & $35.39 \pm 3.57$ & $-2.13 \pm 3.78$ & $18.88 \pm 5.36$ \\
\hline 12. & SPSF012BE & $100.56 \pm 2.86$ & $51.01 \pm 4.30$ & $2.24 \pm 2.13$ & $0.85 \pm 4.99$ \\
\hline 13. & SPSF013BE & $70.74 \pm 2.30$ & $30.45 \pm 3.31$ & $1.08 \pm 2.93$ & $85.14 \pm 2.70$ \\
\hline 14. & SPSF028BE & $99.71 \pm 0.29$ & $123.29 \pm 2.13$ & $87.81 \pm 0.76$ & $92.18 \pm 0.15$ \\
\hline 15. & SPSF029BE & $83.63 \pm 1.89$ & $123.37 \pm 2.04$ & $78.21 \pm 0.78$ & $97.10 \pm 0.18$ \\
\hline 16. & SPSF030BE & $68.62 \pm 7.05^{*}$ & $47.42 \pm 3.37$ & $10.07 \pm 1.76$ & $10.31 \pm 2.43$ \\
\hline 17. & SPSF031BE & $98.55 \pm 2.69$ & $35.87 \pm 1.76$ & $11.89 \pm 1.02$ & $34.92 \pm 2.62$ \\
\hline 18. & SPSF032BE & $98.05 \pm 0.63$ & $21.25 \pm 0.86$ & $11.99 \pm 1.75$ & $12.00 \pm 4.68$ \\
\hline 19. & SPSF033BE & $99.50 \pm 0.09$ & $124.72 \pm 2.27$ & $76.14 \pm 1.16$ & $87.49 \pm 1.15$ \\
\hline 20. & SPSF034BE & $91.26 \pm 0.65$ & $28.02 \pm 2.88$ & $14.27 \pm 2.50$ & $4.43 \pm 1.57$ \\
\hline 21. & SPSF035BE & $99.27 \pm 0.34$ & $35.93 \pm 3.06$ & $14.53 \pm 2.47$ & $7.98 \pm 0.22$ \\
\hline 22. & SPSF037BE & $97.61 \pm 2.82 * *$ & $19.86 \pm 2.06$ & $22.80 \pm 2.69$ & $74.12 \pm 1.64$ \\
\hline 23. & SPSF038BE & $36.72 \pm 1.52$ & $29.12 \pm 2.91$ & $15.58 \pm 1.68$ & $3.40 \pm 3.60$ \\
\hline
\end{tabular}




\begin{tabular}{|c|c|c|c|c|c|}
\hline 24. & SPSF039BE & $76.91 \pm 0.55$ & $31.88 \pm 2.13$ & $15.02 \pm 3.12$ & $5.48 \pm 1.90$ \\
\hline 25. & SPSF040BE & $96.72 \pm 0.82$ & $36.10 \pm 4.11$ & $18.17 \pm 0.53$ & $5.27 \pm 2.84$ \\
\hline 26. & SPSF042BE & $66.24 \pm 4.05$ & $91.71 \pm 5.17$ & $11.45 \pm 2.89$ & $64.38 \pm 4.08$ \\
\hline 27. & SPSF043BE & $96.87 \pm 0.34$ & $81.32 \pm 1.14$ & $7.58 \pm 2.08$ & $24.63 \pm 1.65$ \\
\hline 28. & SPSF044BE & $61.11 \pm 0.51$ & $101.08 \pm 1.85$ & $47.26 \pm 2.26$ & $75.90 \pm 0.76$ \\
\hline 29. & SPSF045BE & $82.56 \pm 0.96$ & $47.38 \pm 2.42$ & $14.09 \pm 3.07$ & $19.99 \pm 2.34$ \\
\hline 30. & SPSF046BE & $82.54 \pm 2.91$ & $36.26 \pm 2.46$ & $17.26 \pm 0.64$ & $2.76 \pm 4.27$ \\
\hline 31. & SPSF047BE & $91.51 \pm 0.68$ & $67.22 \pm 1.75$ & $15.86 \pm 2.28$ & $17.17 \pm 2.44$ \\
\hline 32. & SPSF048CE & $88.92 \pm 1.42$ & $45.87 \pm 2.66$ & $15.37 \pm 1.10$ & $1.48 \pm 1.96$ \\
\hline 33. & SPSF049BW & $54.69 \pm 3.32$ & $100.73 \pm 2.93$ & $16.54 \pm 0.58$ & $73.93 \pm 0.94$ \\
\hline 34. & SPSF051BW & $91.44 \pm 2.23$ & $95.85 \pm 3.58$ & $47.76 \pm 2.91$ & $95.54 \pm 3.12$ \\
\hline 35. & SPSF206CM & $17.98 \pm 2.89$ & $29.19 \pm 3.30$ & $20.58 \pm 2.08$ & $10.60 \pm 1.28$ \\
\hline 36. & SPSF206BE & $79.43 \pm 1.19$ & $66.72 \pm 1.47$ & $12.77 \pm 2.51$ & $47.12 \pm 1.08$ \\
\hline 37. & SPSF208BE & $98.31 \pm 1.19$ & $49.56 \pm 1.84$ & $3.63 \pm 3.35$ & $30.30 \pm 3.79$ \\
\hline 38. & SPSF208CE & $56.45 \pm 2.57$ & $87.78 \pm 4.80$ & $47.18 \pm 0.96$ & $69.91 \pm 2.07$ \\
\hline 39. & SPSF208CH & $69.61 \pm 0.79$ & $53.48 \pm 0.87$ & $8.58 \pm 5.15$ & $31.94 \pm 1.39$ \\
\hline 40. & SPSF209CE & $60.34 \pm 1.41$ & $56.99 \pm 0.99$ & $4.97 \pm 5.9$ & $25.51 \pm 1.83$ \\
\hline 41. & SPSF209BE & $84.76 \pm 2.89$ & $30.29 \pm 5.10$ & $14.50 \pm 2.98$ & $19.36 \pm 1.96$ \\
\hline 42. & SPSF209BW & $27.98 \pm 3.35$ & $23.05 \pm 2.55$ & $15.63 \pm 2.04$ & $3.10 \pm 2.20$ \\
\hline 43. & SPSF211CE & $81.43 \pm 3.26$ & $43.32 \pm 5.68$ & $17.42 \pm 2.90$ & $7.63 \pm 1.68$ \\
\hline 44. & SPSF211BE & $101.26 \pm 1.73$ & $-26.87 \pm 3.20$ & $11.987 \pm 4.55$ & $9.84 \pm 3.43$ \\
\hline 45. & SPSF213BE & $64.55 \pm 0.98$ & $32.73 \pm 2.66$ & $11.35 \pm 1.45$ & $1.12 \pm 2.28$ \\
\hline 46. & SPSF219BE & $98.29 \pm 3.64$ & $72.42 \pm 1.29$ & $8.92 \pm 1.74$ & $61.34 \pm 0.95$ \\
\hline 47. & SPSF221CE & $27.00 \pm 2.80$ & $69.44 \pm 2.22$ & $54.79 \pm 1.63$ & $86.15 \pm 1.03$ \\
\hline 48. & SPSF221CH & $18.90 \pm 1.20$ & $78.33 \pm 1.48$ & $59.95 \pm 3.02$ & $89.21 \pm 1.18$ \\
\hline 49. & SPSF221BE & $89.86 \pm 4.55$ & $81.60 \pm 1.59$ & $41.67 \pm 1.99$ & $34.61 \pm 1.29$ \\
\hline 50. & SPSF224BE & $104.83 \pm 3.40$ & $76.13 \pm 2.25$ & $15.84 \pm 1.85$ & $46.84 \pm 2.28$ \\
\hline 51. & SPSF224CE & $100.00 \pm 00$ & $91.07 \pm 2.32$ & $22.83 \pm 1.79$ & $76.62 \pm 1.39$ \\
\hline 52. & SPSF225CE & $51.23 \pm 1.28$ & $52.13 \pm 4.25$ & $16.59 \pm 3.59$ & $53.98 \pm 0.97$ \\
\hline 53. & SPSF225BE & $88.02 \pm 1.71$ & $-2.74 \pm 2.57$ & $6.72 \pm 2.72$ & $19.33 \pm 1.50$ \\
\hline 54. & SPSF226BE & $86.91 \pm 1.94$ & $55.37 \pm 1.36$ & $18.10 \pm 1.13$ & $40.49 \pm 2.47$ \\
\hline 55. & SPSF226CE & $54.70 \pm 4.90$ & $55.82 \pm 2.54$ & $5.38 \pm 2.55$ & $28.49 \pm 1.82$ \\
\hline 56. & SPSF226CH & $29.86 \pm 3.83$ & $47.69 \pm 1.43$ & $16.99 \pm 1.99$ & $30.81 \pm 1.64$ \\
\hline 57. & SPSF227BE & $57.93 \pm 1.11$ & $59.60 \pm 2.51$ & $9.87 \pm 3.11$ & $35.95 \pm 2.81$ \\
\hline 58. & SPSF227CE & $18.55 \pm 0.16$ & $79.97 \pm 1.55$ & $3.58 \pm 0.07$ & $43.53 \pm 1.70$ \\
\hline 59. & SPSF227BW & $91.04 \pm 0.19$ & $21.39 \pm 3.31$ & $17.16 \pm 2.36$ & $0.29 \pm 1.10$ \\
\hline 60. & SPSF234CE & $100.32 \pm 0.36$ & $17.71 \pm 2.50$ & $18.94 \pm 2.47$ & $33.28 \pm 1.01$ \\
\hline 61. & SPSF234BE & $99.97 \pm 1.33$ & $7.83 \pm 2.73$ & $13 . .57 \pm 3.93$ & $21.58 \pm 3.44$ \\
\hline 62. & SPSF234BW & $74.81 \pm 2.17$ & $21.58 \pm 3.09$ & $18.51 \pm 2.54$ & $-5.90 \pm 1.40$ \\
\hline 63. & SPSF236BE & $61.00 \pm 2.34$ & $45.59 \pm 1.35$ & $16.54 \pm 5.70$ & $27.39 \pm 1.34$ \\
\hline 64. & SPSF236CE & $52.05 \pm 2.73$ & $55.46 \pm 4.41$ & $15.75 \pm 2.32$ & $31.16 \pm 2.54$ \\
\hline 65. & SPSF238CE & $54.05 \pm 1.68$ & $48.16 \pm 1.83$ & $22.35 \pm 2.05$ & $51.68 \pm 1.10$ \\
\hline 66. & SPSF240BE & $93.63 \pm 2.19$ & $37.60 \pm 0.75$ & $3.44 \pm 2.64$ & $14.99 \pm 2.80$ \\
\hline 67. & SPSF240CE & $62.57 \pm 3.85$ & $68.24 \pm 1.09$ & $7.45 \pm 3.44$ & $60.05 \pm 2.44$ \\
\hline
\end{tabular}




\begin{tabular}{|c|c|c|c|c|c|}
\hline 68. & SPSF240CH & $77.42 \pm 4.69$ & $84.38 \pm 2.40$ & $16.16 \pm 2.69$ & $67.47 \pm 1.55$ \\
\hline 69. & SPSF241BE & $85.43 \pm 0.48$ & $65.92 \pm 1.54$ & $11.48 \pm 4.19$ & $22.65 \pm 2.12$ \\
\hline 70. & SPSF244CE & $44.84 \pm 1.14$ & $88.40 \pm 2.66$ & $22.00 \pm 5.72$ & $74.00 \pm 0.80$ \\
\hline 71. & SPSF244BE & $51.29 \pm 1.87$ & $27.26 \pm 2.09$ & $7.28 \pm 0.22$ & $16.22 \pm 3.81$ \\
\hline 72. & SPSF248CH & $82.27 \pm 2.24$ & $41.81 \pm 189$ & $0.01 \pm 5.91$ & $19.57 \pm 3.80$ \\
\hline 73. & SPSF248CE & $69.61 \pm 0.79$ & $95.63 \pm 2.32$ & $-4.28 \pm 5.95$ & $61.16 \pm 1.78$ \\
\hline 74. & SPSF248BE & $86.91 \pm 1.94$ & $103.34 \pm 1.94$ & $35.60 \pm 0.45$ & $81.46 \pm 1.08$ \\
\hline 75. & SPSF250BE & $103.70 \pm 2.01$ & $56.98 \pm 4.65$ & $55.96 \pm 0.61$ & $3.42 \pm 1.73$ \\
\hline 76. & SPSF304BE & $84.80 \pm 0.74$ & $82.98 \pm 1.31$ & $8.60 \pm 3.55$ & $59.79 \pm 4.21$ \\
\hline 77. & SPSF304CH & $100.00 \pm 00$ & $92.93 \pm 2.39$ & $17.96 \pm 2.27$ & $69.50 \pm 1.85$ \\
\hline 78. & SPSF304BW & $52.97 \pm 1.23$ & $51.44 \pm 3.15$ & $20.26 \pm 1.69$ & $6.56 \pm 3.73$ \\
\hline 79. & SPSF310BE & $119.00 \pm 2.91$ & $40.05 \pm 2.46$ & $-24.76 \pm 6.66$ & $15.08 \pm 2.77$ \\
\hline 80. & SPSF310CE & $67.96 \pm 2.27$ & $71.33 \pm 3.08$ & $-7.60 \pm 7.98$ & $20.42 \pm 1.30$ \\
\hline 81. & SPSF310BW & $84.37 \pm 2.21$ & $62.61 \pm 1.34$ & $20.82 \pm 3.09$ & $11.83 \pm 4.56$ \\
\hline 82. & SPSF312BE & $85.73 \pm 1.09$ & $85.23 \pm 2.13$ & $15.40 \pm 2.22$ & $86.36 \pm 0.88$ \\
\hline 83. & SPSF312CE & $79.36 \pm 1.75$ & $80.29 \pm 5.31$ & $26.00 \pm 2.61$ & $64.01 \pm 2.36$ \\
\hline 84. & SPSF318BE & $53.42 \pm 1.51$ & $53.16 \pm 1.44$ & $2.73 \pm 2.20$ & $21.58 \pm 2.80$ \\
\hline 85. & SPSF318CE & $53.04 \pm 1.44$ & $84.00 \pm 2.30$ & $18.05 \pm 1.50$ & $84.27 \pm 1.10$ \\
\hline 86. & SPSF325BE & $100.00 \pm 00$ & $25.83 \pm 1.72$ & $4.16 \pm 1.89$ & $70.96 \pm 4.20$ \\
\hline 87. & SPSF325CE & $100.00 \pm 00$ & $25.98 \pm 3.36$ & $17.27 \pm 2.27$ & $34.52 \pm 2.06$ \\
\hline 88. & SPSF325CH & $100.00 \pm 00$ & $31.76 \pm 6.15$ & $22.57 \pm 1.83$ & $17.53 \pm 3.26$ \\
\hline 89. & SPSF328BE & $100.00 \pm 00$ & $38.77 \pm 2.39$ & $4.96 \pm 3.75$ & $31.69 \pm 4.20$ \\
\hline 90. & SPSF329BE & $86.27 \pm 3.19$ & $27.53 \pm 2.42$ & $13.96 \pm 3.06$ & $-2.60 \pm 1.63$ \\
\hline 91. & SPSF329CE & $100.00 \pm 00$ & $-0.92 \pm 2.54$ & $20.46 \pm 5.41$ & $78.68 \pm 1.47$ \\
\hline 92. & SPSF329CH & $100.00 \pm 00$ & $10.69 \pm 1.47$ & $35.15 \pm 4.35$ & $55.29 \pm 2.21$ \\
\hline 93. & SPSF330BE & $55.29 \pm 2.57$ & $61.32 \pm 1.49$ & $16.57 \pm 1.97$ & $36.87 \pm 4.10$ \\
\hline 94. & SPSF330CE & $98.49 \pm 1.23$ & $37.64 \pm 1.56$ & $11.59 \pm 1.39$ & $15.18 \pm 1.16$ \\
\hline 95. & SPSF339BE & $94.35 \pm 0.69$ & $105.03 \pm 0.62$ & $57.78 \pm 2.05$ & $85.23 \pm 0.23$ \\
\hline 96. & SPSF339CE & $96.87 \pm 0.60$ & $98.44 \pm 0.57$ & $16.25 \pm 3.11$ & $24.43 \pm 2.10$ \\
\hline 97. & SPSF339BW & $51.24 \pm 0.25$ & $70.23 \pm 1.10$ & $3.53 \pm 2.59$ & $78.80 \pm 1.50$ \\
\hline 98. & SPSF347BE & $77.16 \pm 2.57$ & $39.60 \pm 3.54$ & $16.07 \pm 1.66$ & $24.57 \pm 3.57$ \\
\hline 99. & SPSF358BW & $83.21 \pm 3.35$ & $23.88 \pm 2.73$ & $10.81 \pm 1.03$ & $-2.36 \pm 1.44$ \\
\hline 100. & SPSF358BE & $59.77 \pm 3.95$ & $9.41 \pm 1.05$ & $9.31 \pm 0.86$ & $10.20 \pm 1.39$ \\
\hline 101. & SPSF358CE & $98.90 \pm 3.72$ & $28.65 \pm 1.01$ & $5.22 \pm 4.23$ & $16.98 \pm 2.24$ \\
\hline 102. & SPSF360BE & $32.08 \pm 0.15$ & $105.04 \pm 0.89$ & $72.32 \pm 0.71$ & $97.33 \pm 0.10$ \\
\hline 103. & SPSF360CH & $13.12 \pm 4.26$ & $105.22 \pm 1.07$ & $89.32 \pm 0.29$ & $98.90 \pm 0.20$ \\
\hline \multirow[t]{4}{*}{104.} & SPSF360BW & $64.63 \pm 1.57$ & $30.96 \pm 1.57$ & $27.93 \pm 1.43$ & $6.74 \pm 1.61$ \\
\hline & Acarbose & $86.87 \pm 0.66$ & - & - & - \\
\hline & Camptothecin & & & & $94.01 \pm 0.17$ \\
\hline & Indomethacin & & $61.08 \pm 0.68$ & $25.42 \pm 2.99$ & \\
\hline
\end{tabular}




\section{PRELIMINARY QUALITATIVE CHEMICAL SCREENING OF P. maximae EXTRACTS}

The qualitative metabolite analysis of $P$. maximae extracts was obtained as shown in Table 2. The results showed that tannin and alkaloid were present in all the crude extracts while cardiac glycoside, steroid, saponin, and terpenoid were absent. Flavonoid was found in cell ethyl acetate extract (CE), broth ethyl acetate extract $(\mathrm{BE})$, and cell hexane extract $(\mathrm{CH})$. Coumarin and anthraquinone were absent in cell ethyl acetate extract (CE).
This result showed that $P$. maximae produced various types of secondary metabolites. Moreover, the data were in accordance with previous reports that alkaloids, tannins, flavonoids, coumarins and anthraquinones had been found in Penicillium genus. Alkaloids were found in P. aurantiogriseum, P. terlikowskii, and $P$. citrinum, respectively (Kalinina et al. 2018; Lai et al. 2013; Waring et al. 1987). Tannins were found in P. frequentans (Bhardwaj et al. 2015). Flavonoids, coumarins and anthraquinones were obtained from $P$. setosum (George et al. 2019), P. oxalicum (Wang et al. 2014), and P. chrysogenum (Brunati et al. 2009).

TABLE 2. Chemical screening of $P$. maximae

\begin{tabular}{lccccc}
\hline \multirow{2}{*}{ Chemicals } & \multicolumn{3}{c}{ Extracts* } & & CH \\
\cline { 2 - 5 } & CE & BE & BW & CM & $/$ \\
\hline Tannin & $/$ & $/$ & $/$ & $/$ & $/$ \\
Alkaloid & $/$ & $/$ & $/$ & X & X \\
Cardiac glycoside & $\mathrm{X}$ & $\mathrm{X}$ & $\mathrm{X}$ & $\mathrm{X}$ & $\mathrm{X}$ \\
Steroid & $\mathrm{X}$ & $\mathrm{X}$ & $\mathrm{X}$ & $\mathrm{X}$ & $\mathrm{X}$ \\
Saponin & $\mathrm{X}$ & $\mathrm{X}$ & $\mathrm{X}$ & $\mathrm{X}$ & $/$ \\
Flavonoid & $/$ & $/$ & $\mathrm{X}$ & $\mathrm{X}$ & $\mathrm{X}$ \\
Triterpenoid & $\mathrm{X}$ & $\mathrm{X}$ & $\mathrm{X}$ & $\mathrm{X}$ & $\mathrm{X}$ \\
Coumarin & $/$ & $\mathrm{X}$ & $\mathrm{X}$ & $\mathrm{X}$ & $\mathrm{X}$ \\
Anthraquinone & $/$ & $\mathrm{X}$ & $\mathrm{X}$ & & \\
\hline
\end{tabular}

$I=$ Present,$X=$ Absent

*CE: cell ethyl acetate extract; BE: broth ethyl acetate extract; BW: broth water extract; $\mathrm{CM}$ : cell methanol extract; $\mathrm{CH}$ : cell hexane extract

\section{SECONDARY QUANTITATIVE CHEMICAL SCREENING OF P. maximae EXTRACTS}

DETERMINATION OF TOTAL PHENOLIC CONTENT

The total phenolic content of $P$. maximae extracts were shown in Table 3. Broth water extract (BW) and cell methanol extract $(\mathrm{CM})$ showed low amounts of total phenolic content at $1.83 \pm 0.41$ and $0.97 \pm 0.20 \mathrm{mg} \mathrm{GAE} / \mathrm{g}$ dry weight of sample, respectively. Meanwhile, cell ethyl acetate extract (CE) contained the highest amount of phenolic content up to $8.89 \pm 0.40 \mathrm{mg}$ GAE/g dry weight of sample.

\section{DETERMINATION OF TOTAL PHENOLIC CONTENT}

Total flavonoid content of $P$. maximae extracts quantified using the aluminum chloride colorimetric method showed that the total flavonoid content was highest in CE (7.73 mg QE/g dry w.), while flavonoid was absent in BW and CM (Table 3). The maximum phenolic and flavonoid 
content were found in $\mathrm{CE}$ at $8.89 \pm 0.40 \mathrm{mg} \mathrm{GAE} / \mathrm{g}$ and $7.73 \mathrm{mg} \mathrm{QE} / \mathrm{g}$, respectively. The results suggested that ethyl acetate was the most suitable solvent to isolate $P$. maximae secondary metabolites. This may be due to the low polarity property of ethyl acetate which selectively extracts low molecular weight phenol and flavonoid (Bhardwaj et al. 2015). Phenolic and flavonoid are major secondary metabolites of fungi and have been associated with antioxidative action in biological systems (Devi et al. 2012). The presence of phenolic and flavonoid compounds has been reported in Penicillium species including P. chrysogenum (Sikandar et al. 2020), $P$. granulatum (Chandra \& Arora 2012), and P. fumiculosum (Jakovljević et al. 2014). Therefore, the phenolic and flavonoid compounds found in this study possibly possess antioxidant activity.

TABLE 3. Total phenolic and flavonoid contents of $P$. maximae extracts

\begin{tabular}{lcccc}
\hline Extracts* & $\begin{array}{c}\text { Total phenolic content } \\
\text { GAE } / \mathrm{g})\end{array}$ & $\mathrm{mg}$ & Total flavonoid content $(\mathrm{mg}$ QE $/ \mathrm{g})$ & $\mathrm{SD}$ \\
\hline $\mathrm{CE}$ & 8.89 & 0.40 & 7.73 & 0.19 \\
$\mathrm{BE}$ & 6.12 & 0.20 & 2.24 & 0.15 \\
$\mathrm{BW}$ & 1.83 & 0.41 & $\mathrm{n} / \mathrm{d}^{* *}$ & $\mathrm{n} / \mathrm{d}^{* *}$ \\
$\mathrm{CM}$ & 0.97 & 0.20 & $\mathrm{n} / \mathrm{d}^{* *}$ & $\mathrm{n} / \mathrm{d}^{* *}$ \\
$\mathrm{CH}$ & 5.33 & 0.40 & 1.36 & 0.18 \\
\hline
\end{tabular}

${ }^{*} \mathrm{CE}$ : cell ethyl acetate extract; BE: broth ethyl acetate extract; BW: broth water extract; CM: cell methanol extract; $\mathrm{CH}$ : cell hexane extract

\section{CHEMICAL PROFILING OF CELL HEXANE EXTRACT OF $P$. maximae}

From this investigation, $\mathrm{CH}$ of $P$. maximae showed the highest activity of alpha-glucosidase inhibition. Thus, $\mathrm{CH}$ was selected for further analysis. The GCMS chromatogram showed three dominant peaks at retention times of 15.9212, 26.6168, and $37.8967 \mathrm{~min}$, respectively (Figure 1; Table 4). These peaks represented the $n$-Hexadecanoic acid $\left(\mathrm{C}_{16} \mathrm{H}_{32} \mathrm{O}_{2}\right), 2,6$-dimethyl-N(2-methyl-alpha-phenylbenzyl) aniline $\left(\mathrm{C}_{22} \mathrm{H}_{23} \mathrm{~N}\right)$ and 3 Beta-acetoxy-6-nitroandrost-5-en-17-one $\left(\mathrm{C}_{21} \mathrm{H}_{29} \mathrm{NO}_{5}\right)$, respectively. From the previous report (Artanti et al. 2012), n-Hexadecanoic acid had a low activity of alphaglucosidase inhibition. Therefore, alpha-glucosidase inhibition potency of $\mathrm{CH}$ might be from 2,6-dimethyl$\mathrm{N}$-(2-methyl-alpha-phenylbenzyl) aniline $\left(\mathrm{C}_{22} \mathrm{H}_{23} \mathrm{~N}\right)$ and 3Beta-acetoxy-6-nitroandrost-5-en-17-one $\left(\mathrm{C}_{21} \mathrm{H}_{29} \mathrm{NO}_{5}\right)$. However, the anti-alpha-glucosidase efficacy of these two compounds should be further studied to determine whether the anti-alpha-glucosidase capacity of $\mathrm{CH}$ possibly was due to the effect of each individual constituent or the synergistic action of the components in the extract.

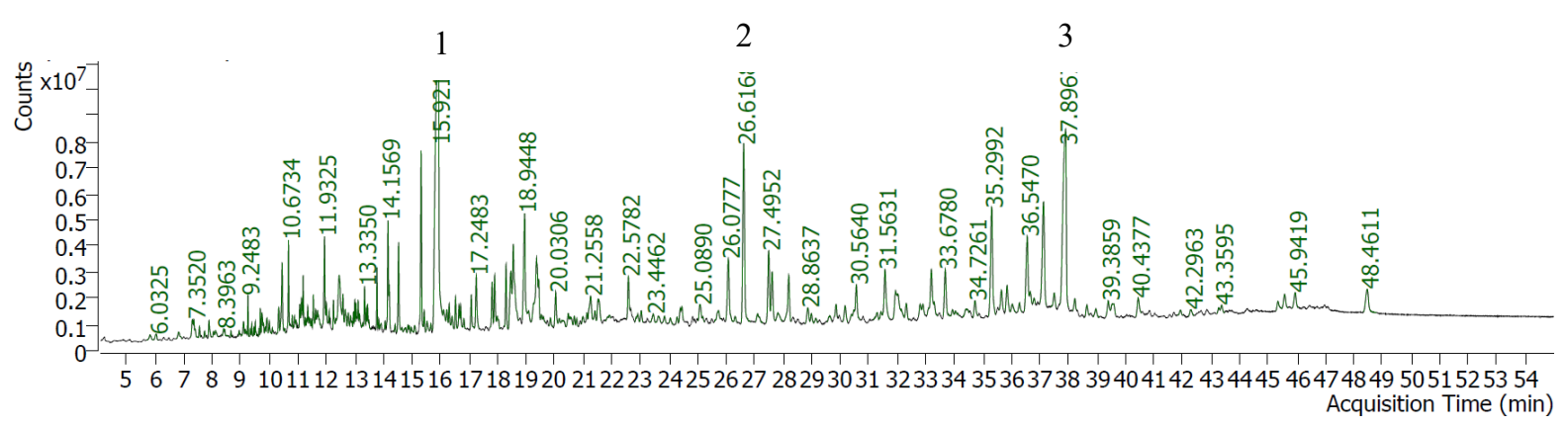

FIGURE 1. GC-MS chromatogram of cell hexane extract of $P$. maximae 
TABLE 4. Dominant peaks from GC-MS chromatogram of cell hexane extract of $P$. maximae

\begin{tabular}{|c|c|c|c|c|c|c|}
\hline No. & Component RT & Compound name & CAS\# & Formula & $\begin{array}{l}\text { Component } \\
\text { Area }\end{array}$ & $\begin{array}{l}\text { Match } \\
\text { factor }\end{array}$ \\
\hline 1 & 15.9212 & n-Hexadecanoic acid & $57-10-3$ & $\mathrm{C}_{16} \mathrm{H}_{32} \mathrm{O}_{2}$ & 99116607.0 & 96.4 \\
\hline 2 & 26.6168 & $\begin{array}{l}\text { 2,6 dimethyl-N-(2 methyl- } \\
\text { alpha.- phenylbenzyl)aniline }\end{array}$ & 119971-00-5 & $\mathrm{C}_{22} \mathrm{H}_{23} \mathrm{~N}$ & 31006746.8 & 81.4 \\
\hline 3 & 37.8967 & $\begin{array}{c}\text { 3Beta-acetoxy-6-nitroandrost- } \\
\text { 5-en-17-one }\end{array}$ & $31559-86-1$ & $\mathrm{C}_{21} \mathrm{H}_{29} \mathrm{NO}_{5}$ & 54667640.7 & 71.4 \\
\hline
\end{tabular}

\section{CHEMICAL PROFILING OF SEMI-PURIFIED PM1}

GC-MS analysis of semi-purified PM1 was shown in Figure 2. The chromatogram illustrated two dominant peaks at retention times (RT) of 14.1728 and 12.3793 min, respectively (Table 5). The highest peak (RT 14.1728) represented 3-(1-methylbutyl)-1,2,4- cyclopentanetrione $\left(\mathrm{C}_{10} \mathrm{H}_{14} \mathrm{O}_{3}\right)$ and the other (RT 12.3793) represented suberic dihydrazide $\left(\mathrm{C}_{8} \mathrm{H}_{18} \mathrm{~N}_{4} \mathrm{O}_{2}\right)$. Their alphaglucosidase inhibitory efficacies have not been reported yet. To the best of our knowledge, the exact active substances of semi-purified PM1 on anti-alpha-glucosidase are still undefined. Thus, anti-alpha-glucosidase efficacy of these two ingredients should be further evaluated.

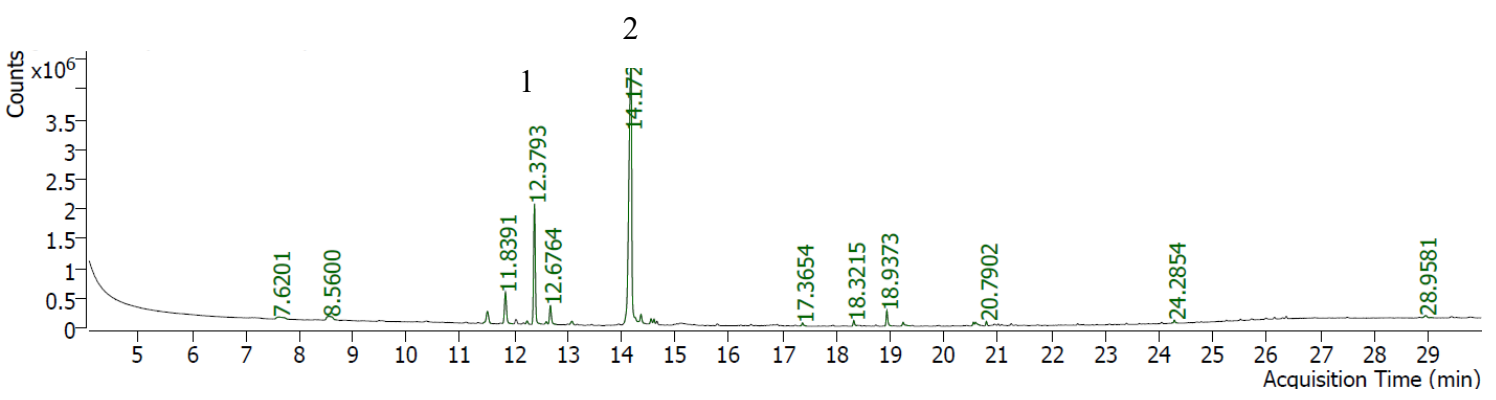

FIGURE 2. GC-MS chromatogram of semi-purified PM1

TABLE 5. Dominant peaks from GC-MS chromatogram of semi-purified PM1

\begin{tabular}{|c|c|c|c|c|c|c|}
\hline No. & Component RT & Compound name & CAS\# & Formula & $\begin{array}{c}\text { Component } \\
\text { area }\end{array}$ & $\begin{array}{l}\text { Match } \\
\text { factor }\end{array}$ \\
\hline 1 & 12.3793 & Suberic dihydrazide & $20247-84-1$ & $\mathrm{C}_{8} \mathrm{H}_{18} \mathrm{~N}_{4} \mathrm{O}_{2}$ & 4511271 & 68.4 \\
\hline 2 & 14.1728 & $\begin{array}{l}\text { 3-(1-methylbutyl)-1,2,4- } \\
\text { cyclopentanetrione }\end{array}$ & $54644-19-8$ & $\mathrm{C}_{10} \mathrm{H}_{14} \mathrm{O}_{3}$ & 16922005 & 82.3 \\
\hline
\end{tabular}




\section{BIOACTIVITIES OF P. maximae ALPHA-GLUCOSIDASE INHIBITORY ACTIVITY}

$P$. maximae extracts were determined for alphaglucosidase inhibition and the half maximal inhibitory concentration $\left(\mathrm{IC}_{50}\right)$. The results showed that $\mathrm{CH}$ was the most active extract for alpha-glucosidase inhibition with $\mathrm{IC}_{50}$ of $490 \mu \mathrm{g} / \mathrm{mL}$ whereas acarbose as standard drug showed the $\mathrm{IC}_{50}$ value at $205 \mu \mathrm{g} / \mathrm{mL}$ (Table 6). The comparison of alpha-glucosidase inhibition from all SPSF224 extracts, CH, CE, and BE showed that their alpha-glucosidase inhibitory activities were higher than the others. Previous studies have reported that phenolic and flavonoid compounds could act as alpha-glucosidase inhibitors and regulators of hyperglycemia (MontefuscoPereira et al. 2013). Therefore, phenolic and flavonoid compounds present in $\mathrm{CH}, \mathrm{CE}$, and $\mathrm{BE}$ could be the chemical groups in these extracts that exhibited the alpha-glucosidase inhibition.

TABLE 6 . The alpha-glucosidase inhibitory activity of $P$. maximae extracts

\begin{tabular}{lcc}
\hline Extracts/Compound & Anti-alpha-glucosidase activity $(\%$ inhibition $)$ & $\mathrm{IC}_{50}(\mu \mathrm{g} / \mathrm{mL})$ \\
\hline Broth ethyl acetate (BE) & $97.12 \pm 1.01$ & 1008 \\
Broth water (BW) & $45.63 \pm 0.21$ & - \\
Cell ethyl acetate (CE) & $118.59 \pm 2.71$ & 490 \\
Cell hexane (CH) & $104.03 \pm 0.42$ & - \\
Cell methanol (CM) & $27.77 \pm 0.11$ & $-*$ \\
Semi-purified PM1 & $91.83 \pm 1.59$ & 205 \\
Acarbose &
\end{tabular}

Data were expressed as mean $\pm \mathrm{SD}$

$\mathrm{p}=$ Positive control

* = insufficient amount

\section{ANTIOXIDANT ACTIVITY}

Antioxidant activities of $P$. maximae extracts were determined by the DPPH radical scavenging activity. The highest activity of antioxidant was found in CE. However, its ability was less than a standard quercetin (Table 7). Huang et al. (2005) reported that phenolic compounds were the major antioxidant constituents of the endophytes. In addition, previous report showed the correlation between the phenolic and flavonoid contents with antioxidant activity (Shweta et al. 2010). In this study, CE exerted highest DPPH radical scavenging activity. Hence, it was possibly due to the highest level of phenolic and flavonoid contents in the extract.

TABLE 7. Inhibition of DPPH radical scavenging activity for $P$. maximae extracts

\begin{tabular}{ccc}
\hline Samples* & \% inhibition at $200 \mu \mathrm{g} / \mathrm{mL}^{* *}$ & $\mathrm{SD}$ \\
\hline $\mathrm{CE}$ & 31.25 & 5.89 \\
$\mathrm{BE}$ & 28.40 & 2.39 \\
$\mathrm{BW}$ & 2.66 & 0.33 \\
$\mathrm{CM}$ & 1.47 & 0.26 \\
$\mathrm{CH}$ & 11.69 & 2.14 \\
quercetin & 93.44 & 1.54 \\
\hline
\end{tabular}

*CE: cell ethyl acetate extract; BE: broth ethyl acetate extract; BW: broth water extract; CM: cell methanol extract; $\mathrm{CH}$ : cell hexane extract.

**Values were represented with mean \pm standard deviation of three replications $(\mathrm{n}=3)$ 


\section{ANTIMICROBIAL ACTIVITY}

Crude extracts of $P$. maximae were tested for antimicrobial activity using the disc diffusion method. Broth ethyl acetate extract (BE) showed inhibition zone against $S$. aureus, T. mentagrophytes, and T. rubrum at $8.1 \pm 0.1$, $6.4 \pm 0.5$, and $11.2 \pm 2.6 \mathrm{~mm}$, respectively. Cell methanol extract (CM) also exhibited inhibition against T. rubrum at $12.6 \pm 3.5 \mathrm{~mm}$. However, all extracts did not inhibit the Gram-negative bacteria, E. coli, $P$. aeruginosa, and yeast (C. albicans) (Table 8). This study was consistent with previous report that the extracts from Penicillium species also have the antibacterial potency on Gram positive bacteria including griseofulvin from $P$. brasilianum (Tang et al. 2015) and scleroderolide from Penicillium sp. FO-5637 (Tomoda et al. 1998). The result suggested that the extracts were potent in killing Gram-positive bacteria. Nevertheless, the extracts from other Penicillium species such as P. Janthinellum (Do Rosário Marinho et al. 2005) and P. herquei (Marinho et al. 2013) possessed bactericidal activity on Gram negative bacteria.

TABLE 8 . Anti-microbial activity of $P$. maximae

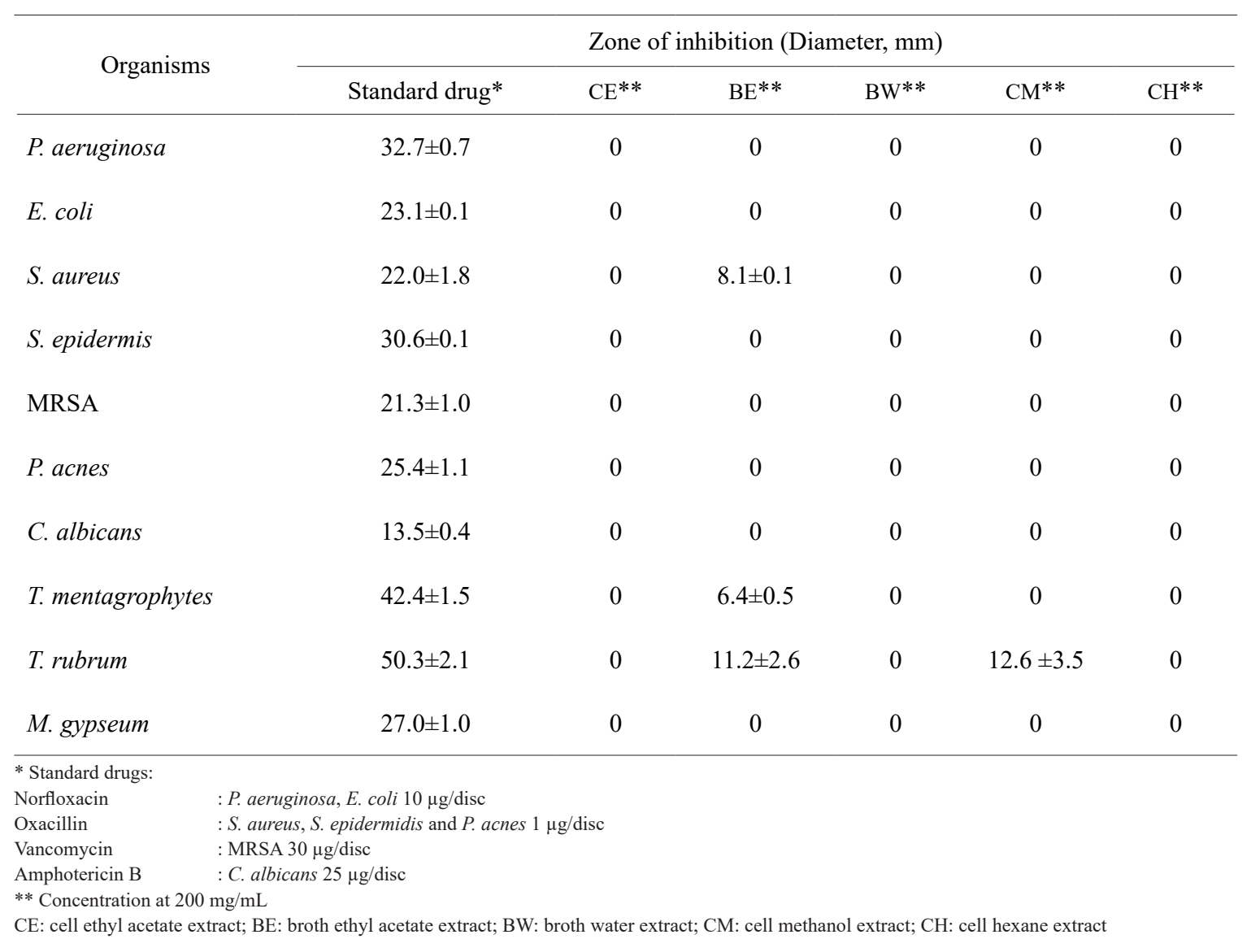

\section{CONCLUSION}

Fifty-four soil fungi were collected from Sirindhorn peat swamp forest in Narathiwat Province, Thailand and 104 crude extracts were obtained from them. SPSF224 was selected for further study because of the potential effects on biological activities. SPSF224 was identified by molecular method as $P$. maximae. From phytochemical investigation, its crude extracts contained a variety of 
secondary metabolites including tannins, alkaloids, flavonoids, coumarins and anthraquinones. P. maximae extracts also showed high potential effect on alphaglucosidase inhibition and moderate potential effect on antimicrobial, anti-inflammation, and antioxidant activities. This study was the first report of chemical investigation and biological activities of the isolated fungus, $P$. maximae. Hence, this information could be used as database for further study of the lead compound for antidiabetic drug from fungi in the future.

\section{ACKNOWLEDGEMENTS}

The authors would like to thank the financial support from the office of the Royal Development Projects Board (ORDPB) under The Royal Initiative of Her Royal Highness Princess Maha Chakri Sirindhorn (RSPG project). The research was also supported by scholarship which supported tuition fees issued by the Discipline of Excellence in Pharmacy Project, The Faculty of Pharmaceutical Sciences, Prince of Songkla University.

\section{REFERENCES}

Artanti, N., Tachibana, S., Kardono, L.B. \& Sukiman, H. 2012. Isolation of alpha-glucosidase inhibitors produced by an endophytic fungus, Colletotrichum sp. TSC13 from Taxus sumatrana. Pakistan Journal of Biological Sciences 15(14): 673-679.

Bischoff, H. 1994. Pharmacology of a-glucosidase inhibition. European Journal of Clinical Investigation 24(3): 3-10.

Bhardwaj, A., Sharma, D., Jadon, N. \& Agrawal, P.K. 2015. Antimicrobial and phytochemical screening of endophytic fungi isolated from spikes of Pinus roxburghii. Archives of Clinical Microbiology 6(3): 1-9.

Boonyuen, N., Sri-Indrasutdhi, V., Suetrong, S., Sivichai, S. \& Jones, E.G. 2012. Annulatascus aquatorba sp. nov., a lignicolous freshwater ascomycete from Sirindhorn peat swamp forest, Narathiwat, Thailand. Mycologia 104(3): 746-757.

Brunati, M., Rojas, J.L., Sponga, F., Ciciliato, I., Losi, D., Göttlich, E. \& Marinelli, F. 2009. Diversity and pharmaceutical screening of fungi from benthic mats of Antarctic lakes. Marine Genomics 2(1): 43-50.

Bureau of Non Communicable Disease (NCD). 2019. The Number of Thais with Diagnosed Diabetes. www.thaincd. com/2016/mission/documents.php. Accessed on December 24, 2019

Casqueiro, J. \& Alves, C. 2012. Infections in patients with diabetes mellitus: A review of pathogenesis. Indian Journal of Endocrinology and Metabolism 16(Supp11): S27.

Cazar, M., Schmeda-Hirschmann, G. \& Astudillo, L. 2005. Antimicrobial butyrolactone I derivatives from the Ecuadorian soil fungus Aspergillus terreus Thorn. var terreus. World Journal of Microbiology and Biotechnology 21(6-7): 10671075.

Chandra, P. \& Arora, D.S. 2012. Optimization of antioxidant potential of Penicillium granulatum Bainier by statistical approaches. International Scholarly Research Notices Microbiology 2012(1): 452024.

Chang, C.C., Yang, M.H., Wen, H.M. \& Chern, J.C. 2002. Estimation of total flavonoid content in propolis by two complementary colorimetric methods. Journal of Food and Drug Analysis 10(3): 178-182.

Dej-adisai, S. \& Pitakbut, T. 2015. Determination of a-glucosidase inhibitory activity from selected Fabaceae plants. Pakistan Journal of Pharmaceutical Sciences 28(5): 1679-1683.

Dej-adisai, S., Phoopha, S. \& Puripattanavong, J. 2018. Phytochemical investigation and bioactivities of Alternanthera ramosissima (Mart.) Chodat and Hassl. Pharmacognosy Magazine 14(57): 346-351.

Deshmukh, S.K., Mishra, P.D., Kulkarni-Almeida, A., Verekar, S., Sahoo, M.R., Periyasamy, G., Goswami, H., Khanna, A., Balakrishnan, A. \& Vishwakarma, R. 2009. Antiinflammatory and anticancer activity of ergoflavin isolated from an endophytic fungus. Chemistry \& Biodiversity 6(5): 784-789.

Devi, N.N., Prabakaran, J.J. \& Wahab, F. 2012. Phytochemical analysis and enzyme analysis of endophytic fungi from Centella asiatica. Asian Pacific Journal of Tropical Biomedicine 2(3): 1280-1284.

Dighton, J. \& White, J.F. 2017. The Fungal Community: Its Organization and Role in the Ecosystem. Boca Raton: CRC Press.

Do Rosário Marinho, A.M., Rodrigues-Filho, E., Maria da Luz, R.M. \& Santos, L.S. 2005. Biologically active polyketides produced by Penicillium janthinellum isolated as an endophytic fungus from fruits of Melia azedarach. Journal of the Brazilian Chemical Society 16(2): 280-283.

Farnsworth, N.R. 1966. Biological and phytochemical screening of plants. Journal of Pharmaceutical Sciences 55(3): 225-276.

Fuchs, P., Perez-Pinzon, M.A. \& Dave, K.R. 2013. Cerebral ischemia in diabetics and oxidative stress. In Diabetes: Oxidative Stress and Dietary Antioxidants. Elsevier Inc. pp. 15-23.

Gao, H., Huang, Y.N., Gao, B., Xu, P.Y., Inagaki, C. \& Kawabata, J. 2008. A-glucosidase inhibitory effect by the flower buds of Tussilago farfara L. Food Chemistry 106(3): 1195-1201.

George, T.K., Devadasan, D. \& Jisha, M.S. 2019. Chemotaxonomic profiling of Penicillium setosum using high-resolution mass spectrometry (LC-Q-ToF-MS). Heliyon 5(9): e02484.

Hakamata, W., Kurihara, M., Okuda, H., Nishio, T. \& Oku, T. 2009. Design and screening strategies for a-glucosidase 
inhibitors based on enzymological information. Current Topics in Medicinal Chemistry 9(1): 3-12.

Harborne, J.B. 1984. Methods of plant analysis. Phytochemical Methods. Dordrecht: Springer. Dordrecht pp. 1-36.

Horii, S., Fukase, H., Matsuo, T., Kameda, Y., Asano, N. \& Matsui, K. 1986. Synthesis and. alpha- $D$-glucosidase inhibitory activity of $\mathrm{N}$-substituted valiolamine derivatives as potential oral antidiabetic agents. Journal of Medicinal Chemistry 29(6): 1038-1046.

Huang, D., Ou, B. \& Prior, R.L. 2005. The chemistry behind antioxidant capacity assays. Journal of Agricultural and Food Chemistry 53(6): 1841-1856.

Jakovljević, V.D., Milićević, J.M., Stojanović, J.D., Solujić, S.R. \& Vrvić, M.M. 2014. Antioxidant activity of ethanolic extract of Penicillium chrysogenum and Penicillium fumiculosum. Hemijska Industrija 68(1): 43-49.

Kalinina, S.A., Kalinin, D.V., Hövelmann, Y., Daniliuc, C.G., Mück-Lichtenfeld, C., Cramer, B. \& Humpf, H.U. 2018. Auranthine, a benzodiazepinone from Penicillium aurantiogriseum: Refined structure, absolute configuration, and cytotoxicity. Journal of Natural Products 81(10): 21772186.

Kalra, S. 2014. Alpha glucosidase inhibitors. The Journal of the Pakistan Medical Association 64(4): 474-476.

Lai, D., Brötz-Oesterhelt, H., Müller, W.E., Wray, V. \& Proksch, P. 2013. Bioactive polyketides and alkaloids from Penicillium citrinum, a fungal endophyte isolated from Ocimum tenuiflorum. Fitoterapia 91: 100-106.

Lorian, V. 2005. Antibiotics in Laboratory Medicine. 5th ed. Philadelphia: Lippincott Williams \& Wilkins.

MacMillan, J. 1954. Griseofulvin. Part IX. Isolation of the bromo-analogue from Penicillium griseofulvum and Penicillium nigricans. Journal of the Chemical Society (Resumed) 1954: 2585-2587.

Marinho, A.M., Marinho, P.S., Santos, L.S., Rodrigues Filho, E. \& Ferreira, I.C. 2013. Active polyketides isolated from Penicillium herquei. Anais da Academia Brasileira de Ciências 85(3): 909-912.

Montefusco-Pereira, C.V., de Carvalho, M.J., de Araújo Boleti, A.P., Teixeira, L.S., Matos, H.R. \& Lima, E.S. 2013. Antioxidant, anti-inflammatory, and hypoglycemic effects of the leaf extract from Passiflora nitida Kunth. Applied Biochemistry and Biotechnology 170(6): 1367-1378.

Office of the Royal Development Projects Board (ORDPB) 2019. Pa Phru to Daeng. www.rdpb.go.th/th. Accessed on September 15, 2019.

Rukachaisirikul, V., Rungsaiwattana, N., Klaiklay, S., Pakawatchai, C., Saithong, S., Phongpaichit, S., Borwornwiriyapan, K. \& Sakayaroj, J. 2013. Indole-benzodiazepine-2, 5-dione derivatives from a soil fungus Aspergillus sp. PSU-RSPG185. Tetrahedron 69(52): 11116-11121.

Sadananda, T., Govindappa, M., Vinay Dutt, G., Bhat, B., Baishya, P. \& Chandrappa, C. 2014. Isolation and characterization of antiviral and ribosome inactivating protein from the endophytic fungi Alternaria sp. from Viscum album using MADLI-TOF-MS and their antibacterial activity. Drug Invention Today 6(2): 105-112.

Schoch, C.L., Seifert, K.A., Huhndorf, S., Robert, V., Spouge, J.L., Levesque, C.A., Chen, W. \& Consortium, F.B. 2012. Nuclear ribosomal internal transcribed spacer (ITS) region as a universal DNA barcode marker for fungi. Proceedings of the National Academy of Sciences of the United States of America 109(16): 6241-6246.

Scott, J.A. \& King, G.L. 2004. Oxidative stress and antioxidant treatment in diabetes. Annals of the New York Academy of Sciences 1031(1): 204-213.

Shwab, E.K. \& Keller, N.P. 2008. Regulation of secondary metabolite production in filamentous ascomycetes. Mycological Research 112(2): 225-230.

Shweta, S., Ritesh, T., Khadabadi, S.S. \& Deokate, U.A. 2010. In vitro antioxidant activity and total phenolic, flavonoid contents of the crude extracts of Pterospermum acerifolium Willd leaves (Sterculiaceae). Journal of Chemical and Pharmaceutical Research 2(3): 417-423.

Sikandar, A., Zhang, M., Wang, Y., Zhu, X., Liu, X., Fan, H. \& Duan, Y. 2020. Mycochemical screening and analysis, antioxidant activity, and biochemical composition of fermentation strain Snef1216 (Penicillium chrysogenum). Journal of Analytical Methods in Chemistry 2020(2): 1-8.

Singleton, V.L. \& Rossi, J.A. 1965. Colorimetry of total phenolics with phosphomolybdic-phosphotungstic acid reagents. American Journal of Enology and Viticulture 16(3): 144-158.

Tang, H.Y., Zhang, Q., Li, H. \& Gao, J.M. 2015. Antimicrobial and allelopathic metabolites produced by Penicillium brasilianum. Natural Product Research 29(4): 345-348.

Thawai, C. 2004. Taxonomy of Micromonospora strains from Thai peat swamp forest soils and secondary metabolites of a selected isolate. PhD. Thesis. Chulalongkorn University (Unpublished).

Tomoda, H., Tabata, N., Masuma, R., Si, S.Y. \& Omura, S. 1998. Erabulenols, inhibitors of cholesteryl ester transfer protein produced by Penicillium sp. FO-5637. The Journal of Antibiotics 51(7): 618-623.

Trease, G.E. \& Evans, W.C. 1983. Textbook of Pharmacognosy 12th ed. London: Balliers Tindall. pp. 343-384.

Trisuwan, K., Rukachaisirikul, V., Borwornwiriyapan, K., Phongpaichit, S. \& Sakayaroj, J. 2014. Benzopyranone, benzophenone, and xanthone derivatives from the soil fungus Penicillium citrinum PSU-RSPG95. Tetrahedron Letters 55(7): 1336-1338.

Trisuwan, K., Rukachaisirikul, V., Kaewpet, M., Phongpaichit, S., Hutadilok-Towatana, N., Preedanon, S. \& Sakayaroj, J. 2011. Sesquiterpene and xanthone derivatives from the sea fan-derived fungus Aspergillus sydowii PSU-F154. Journal of Natural Products 74(7): 1663-1667. 
Turbyville, T.J., Wijeratne, E.K., Liu, M.X., Burns, A.M., Seliga, C.J., Luevano, L.A., David, C.L., Faeth, S.H., Whitesell, L. \& Gunatilaka, A.L. 2006. Search for Hsp90 inhibitors with potential anticancer activity: Isolation and SAR studies of radicicol and monocillin I from two plant-associated fungi of the Sonoran desert. Journal of Natural Products 69(2): 178-184.

Visagie, C.M., Houbraken, J., Frisvad, J.C., Hong, S.B., Klaassen, C.H.W., Perrone, G., Seifert, K.A., Varga, J., Yaguchi, T. \& Samson, R.A. 2014. Identification and nomenclature of the genus Penicillium. Studies in Mycology 78: 343-371.

Visagie, C.M., Houbraken, J., Rodriques, C., Pereira, C.S., Dijksterhuis, J., Seifert, K.A., Jacobs, K. \& Samson, R.A. 2013. Five new Penicillium species in section Sclerotiora: A tribute to the Dutch Royal family. Persoonia 31: 42-62.

Wang, P.L., Li, D.Y., Xie, L.R., Wu, X., Hua, H.M. \& Li, Z.L. 2014. Two new compounds from a marine-derived fungus Penicillium oxalicum. Natural Product Research 28(5): 290-293.

Wang, X., You, J., King, J.B., Powell, D.R. \& Cichewicz, R.H. 2012. Waikialoid a suppresses hyphal morphogenesis and inhibits biofilm development in pathogenic Candida albicans. Journal of Natural Products 75(4): 707-715.
Waring, P., Eichner, R.D., Tiwari-Palni, U. \& Mullbacher, A. 1987. Gliotoxin-E: A new biologically-active epipolythiodioxopiperazine isolated from Penicillium terlikowskii. Australian Journal of Chemistry 40(5): 991-997.

World Health Organization (WHO). 2019. Guidelines on Second-and Third-Line Medicines and Type of Insulin for the Control of Blood Glucose Levels in Non-Pregnant Adults with Diabetes Mellitus. http://apps.who.int/iris/bitstream/ handle/10665/272433. Accessed July 7, 2019.

Zhao, J., Li, C., Wang, W., Zhao, C., Luo, M., Mu, F., Fu, Y., Zu, Y. \& Yao, M. 2013. Hypocrea lixii, novel endophytic fungi producing anticancer agent cajanol, isolated from pigeon pea (Cajanus cajan [L.] M illsp.). Journal of Applied Microbiology 115(1): 102-113.

Department of Pharmacognosy and Pharmaceutical Botany Faculty of Pharmaceutical Sciences

Prince of Songkla University

Songkhla 90112

Thailand

*Corresponding author; email: sukanya.d@psu.ac.th

Received: 3 February 2020

Accepted: 6 January 2021 